A SINGLE STAR MODEL FOR V 1016 CYGNI

F.J. Ahern, M.P. FitzGerald, K.A. Marsh, and C.R. Purton Canada Centre for Remote Sensing, University of Waterloo, California Institute of Technology, York University, respectively.

We present a model of the emission object V1016 Cygni consistent with the current optical, infrared and radio observations. V1016 Cygni is $4.2 \pm 1.0 \mathrm{kpc}$ from the sun. It was originally an $\mathrm{M}$ star which ejected $\sim 0.017 \mathrm{M}_{\mathrm{O}}$ at $35 \mathrm{kms}^{-1}$ over a period of $\sim 600$ years producing a neutral nebula with an $\mathrm{r}^{-2}$ density distribution. This process ceased in $1961.3 \pm 3.0$ when the nebula detached from a remnant hot core $\left(\mathrm{T} \approx 80,000 \mathrm{~K}, \mathrm{R} \approx 1.5 \mathrm{R}_{\mathrm{o}}\right)$. By $1964.1 \pm 0.3$ the nebula was ionized by the UV radiation from the core, producing the observed emission line spectrum. The infrared energy excess comes from two dust components of $\sim 1000^{\circ} \mathrm{K}$ and $\sim 250^{\circ} \mathrm{K}$, which absorb UV photons from the star. The dust is concentrated into clumps which 1) provide high density areas on their inner side, allowing strong [OIII] $\lambda 4363$ to remain; 2 ) shield the regions on their outer side permitting low excitation lines to be present; 3) explain the observed emission line structure. A stellar wind of $105 \mathrm{~km} / \mathrm{s}$ helps to keep the centre of the ejected nebula hollow. We suggest V1016 Cygni is an example of a low mass planetary nebula in the formation stage.

OPTICAL HISTORIES OF SOME POSSIBLE EMBRYONIC PLANETARY NEBULAE

P.A. Feldman and C.R. Purton

H.I.A., N.R.C. of Canada, Ottawa and CRESS, York University, Toronto, respectively.

From the list of stellar emission-line objects which exhibit radio spectra characteristic of uniform radial mass outflow, we have selected those objects which might be considered possible embryonic planetary nebulae. Our criterion was to choose those mass outflow objects with exciting stars of the types characteristic of central stars of nonstellar (i.e., normal) planetary nebulae. These objects are Hb 12 , Hen 1044, HD 167362, and possible M2-9, Vy 2-2, and H1-23. 
Recently, Ahern et al. (Astron. and Astrophys. 58, 35 (1977) have argued that V1016 Cygni is a low-mass planetary nebula in the process of formation. It is well-known that V1016 Cygni, the prototypical radioemitting mass outflow object, underwent a 5 mag outburst in 1965 . Therefore, we decided to search for similar optical outbursts from those objects in our short list of possible embryonic planetary nebulae using the archival plate collection of Harvard College Observatory. To our surprise, no large brightenings $(\Delta B>1 \mathrm{mag})$ of the type exhibited by V1016 Cygni were found in the stars associated with other radio-emitting proto-planetary candidates. The implications of this null result for models of the mass outflow from embryonic planetary nebulae will be discussed briefly.

\section{RECOMBINATION LINES FROM COMPACT HII REGIONS}

Peter Silverglate and Yervant Terzian

National Astronomy and Ionosphere Center, Cornell University, Ithaca, N.Y.

Radio recombination lines of hydrogen ( $\mathrm{H} 167 \alpha)$ have been observed from 45 HII regions using the Arecibo telescope. Nine of these sources also show the C167 $\alpha$ line, and twenty show H210B lines. Some of the carbon lines, particularly those from $\mathrm{W} 48$ and $\mathrm{G} 62.2+.5$, show a significant negative displacement from the $H 167 \alpha$ velocities. This can be explained if the lines originate in the ionization front or in neutral material swept up by the ionization front. The displacement is $3.8 \mathrm{~km} / \mathrm{sec}$ for $W 48$, and $5.7 \mathrm{~km} / \mathrm{sec}$ for $\mathrm{G} 62.2+.5$. The source $\mathrm{G} 37.7+.1 \mathrm{ex}-$ hibited two $\mathrm{H} 167 \alpha$ ines, one $25 \mathrm{~km} / \mathrm{sec}$ wide at $87.3 \mathrm{~km} / \mathrm{sec}$ and one $16 \mathrm{~km} / \mathrm{sec}$ wide at $48 \mathrm{~km} / \mathrm{sec}$, velocities with respect to the local standard of rest. This may be a case of two HII regions in the same line of sight. Parameters for the HII regions, such as electron temperatures and densities, are derived. (Paper to appear in the Astronomical Journal.) 